\title{
Manejo de pseudoaneurisma de la arteria esfenopalatina posterior a osteotomía LeFort I
}

\section{Management of a pseudoaneurysm of the sphenopalatine artery following a Le Fort I osteotomy}

Recibido: 15/06/2018 Aceptado: 23/08/2018 Publicado: 01/09/218

\author{
Jorge Barona Terán ${ }^{1}$ \\ Christian Salvador Rodríguez Gómez ${ }^{2}$ \\ Ángel Alvarado Lema $^{3}$ \\ Fernando Sandoval Vernimmen 4
}

1 Instituto de Cirugía Oral y Maxilofacial. José Castillo Mz. 701 V. 22 Kennedy Norte, oficina 1, Casilla postal EC090112. Guayaquil-Ecuador.

Correo electrónico: baronamaxilofacial@yahoo.es

2 Instituto de Cirugía Oral y Maxilofacial. José Castillo Mz. 701 V. 22 Kennedy Norte, oficina 1, Casilla postal EC090112. Guayaquil-Ecuador.

Correo electrónico: salva_8888@hotmail.com

${ }^{3}$ UOMNI Hospital-Guayaquil, Av. Juan Tanca Marengo y Av. Abel Romero Castillo 13E NE, oficina 5 ,

Casilla postal 090513. Guayaquil-Ecuador.

Correo electrónico: angel_alvalema@hotmail.com

4 Universidad San Francisco de Quito, Colegio de Ciencias de la Salud, Escuela de Odontología, Clínica Odontológica, Campus Cumbayá, oficina CO 106, casilla postal 17-1200-841. Quito-Ecuador.

Correo electrónico: achavez@usfq.edu.ec 


\section{Resumen}

La formación de pseudo-aneurismas en el complejo vascular de la región maxilofacial no son comunes, los reportes encontrados en la bibliografía indican que los mecanismos romos (iatrogenia) es la causa principal. Se describe caso de paciente masculino de 17 años de edad con desarmonía dentofacial, que presentó un pseudo-aneurisma de la arteria esfenopalatina derecha, como una rara y seria complicación a una ostetomía Lefort l, misma que se trató con embolización trans-arterial mediante la liberación de dos coils. Se revisó los conceptos de aneurismas, así como las modalidades para su tratamiento. Sugiriendo la intervención endovascular como primera línea de manejo, relegando a la intervención quirúrgica a situaciones de trauma penetrante, con instalaciones y habilidades adecuadas.

Palabras claves: pseudo aneurisma, osteotomía Lefort, cirugía ortognática, Maxilar, embolización, Hemorragía

\section{Abstract}

The formation of pseudo-aneurysms in the vascular complex of the maxillofacial region are not common, reports found in the literature indicate that blunt mechanisms (iatrogenesis) is the main cause. We describe the case of a 17-year-old male patient with dentofacial disharmony, who presented a pseudo-aneurysm of the right sphenopalatine artery, as a rare and serious complication to a Lefort I osteotomy, which was treated with transarterial embolization by means of the release of two coils. We reviewed the concepts of aneurysms as well as the modalities for their treatment. Suggesting endovascular intervention as the first line of management, relegating surgical intervention to situations of penetrating trauma, with adequate facilities and skills.

Keywords: pseudo aneurysm, Lefort osteotomy, orthognathic surgery, Maxilla, embolization, hemorrhage 


\section{Introducción}

Los pseudo-aneurismas pueden formarse en cualquier parte del cuerpo humano, siendo la arteria femoral la más común. Rara vez afectan a la cabeza y cuello, se han reportado que ocurren después de un trauma facial, cirugía ortognática y cirugía de cuello. El $70 \%$ resulta de trauma con objetos romos, $25 \%$ por trauma penetrante y menos de $5 \%$ corresponden a aneurismas extracraneales $\mathbf{1 , 2 , 3}$.

Los aneurismas en la región de cabeza y cuello son escasos, la literatura refiere a los pseudoaneurismas de la carótida externa extremadamente poco comunes, 4 de ellos el más común es la arteria temporal superficial. McCollum reportó una serie de más de 8000 aneurismas, habiendo encontrado sólo 21 pseudo-aneurismas de la carótida externa $\mathbf{5 , 6}$.

Los aneurismas permanecen asintomáticos hasta su ruptura, normalmente un mes posterior a su aparición. Cuando los síntomas se presentan, pueden ir desde rigidez en cuello, dolor, defectos neurológicos, compromiso de la vía aérea, dificultad a la apertura oral, epistaxis, asimetría, déficit de pares craneales y muerte súbita de acuerdo a la localización de la lesión y su origen $1,3,6,7$

Los aneurismas se clasifican en verdaderos, falsos (pseudo-aneurismas) y disecantes.

Los aneurismas verdaderos son el resultado del desgarro o ruptura parcial de la capa muscular e íntima de una arteria; siendo la capa adventicia la que evita una extravasación sanguínea. Su etiología está relacionada con aterosclerosis, sífilis, tumores, y alteraciones congénitas ${ }^{\mathbf{2 , 8}}$.

La transección completa de un lado de una pared arterial conduce a la formación de un pseudoaneurisma o hematoma pulsante que está contenido por los tejidos contiguos. Después de la extravasación de la sangre, el coágulo organizador forma una cápsula pseudofibrosa con una matriz central licuada, resultando en un sangrado adicional y la expansión de la lesión 9,10.

Sin una capa arterial, la presión en el hematoma aumenta hasta que la presión en la zona periarterial se equipara a la presión arterial media. El revestimiento endotelial con frecuencia produce una pseudoíntima continua con el lumen arterial. La licuación del hematoma conduce a la formación de una masa pulsante o soplo que puede romperse, continuar agrandándose o calcificarse. Esta lesión puede no ser evidente porque se encuentra en planos profundos. ${ }^{\mathbf{8}, 9}$

La inestabilidad de la pared del vaso en los aneurismas verdaderos y falsos plantea la amenaza constante de hemorragia o infarto como resultado de un evento tromboembólico. Estas lesiones pueden conducir a estenosis, disección, fístulas arteriovenosas o hemorragias masivas de difícil control. El fenómeno del falso aneurisma puede desarrollarse días, meses o años posteriores al trauma inicial, con complicaciones que van desde asimetrías, defectos neurológicos, hemorragia secundaria y desalojo del trombo. $\mathbf{2 , 3 , 1 0}$

Su etiología es controversial, algunos autores la atribuyen como complicación al borde afilado del complejo pterigoideo durante la fractura y otros a las características anatómicas del paciente en la fístula carotidea 11. En el caso de pseudoaneursmas el origen está ligado entre otras cosas a las heridas por arma de fuego, arma blanca, cerclaje circunmandibular y en cirugías ortognáticas principalmente osteotomías vertical u oblicua mandíbular. Sin embargo, existe evidencia de mayor sangrado posterior a osteotomías en maxilar que puede comprometer la vida del paciente. ${ }^{\mathbf{4} 9}$

Dentro del diagnóstico diferencial tenemos: hematoma, lipoma, quiste de inclusión, cuerpos extraños, angiofibroma, fistula arteriovenosa, neuroma, meningocele y encefalocele. 
El diagnóstico puede ser confirmado con métodos invasivos y no invasivos: ultrasonografía, Ultrasonografía Doppler Color, tomografía computarizada, resonancia magnética, angiografía, sin embargo, la arteriografía es el método de elección. ${ }^{3,11,12,13}$

Están descritos tres distintas formas de tratamiento:

1) Tratamiento conservador. Algunos autores recomiendan terapia con anticoagulantes (ácido acetilsalicílico, clopidogrel),14 esperando la resolución espontánea, sin embargo, es un método asociado a disconfort, dolor y más importante la posibilidad de ruptura del aneurisma, compromiso de vía aérea y erosión ósea a lo largo del tiempo. ${ }^{15,16}$

2) Reparación quirúrgica abierta. El método tradicional va desde la exploración, empaquetamiento, uso de electrocoagulador (diatermia), ligadura de la arteria carótida externa, escisión quirúrgica completa, cierre directo o la colocación de un parche (autólogo o sintético). 9,15,17

3) Embolización percutánea, en la cual se introduce un catéter vía femoral y posterior colocación de stens, coils o balones proximales y distales a la lesión ha mostrado resultados favorables. Cuyo propósito es la estimulación de circulación colateral y no la oclusión de vasos proximales. $\mathbf{1 , 1 0 , 1 8}$

\section{Caso clínico}

En marzo del 2017, se somete a paciente masculino de 17 años de edad, en otro Centro Hospitalario, a cirugía ortognática para corrección de Deformidad Dentofacial (exceso de crecimiento maxilar, incompetencia labial y exposición gingival excesiva), reposicionamiento de maxilar con ostetomía tipo Lefort 1 de intrusión, fijado mediante material de osteosíntesis. A los 15 días del evento quirúrgico desarrolló epistaxis izquierda, por lo que fue ingresado al Servicio de Emergencias, colocando taponamiento nasal anterior y posterior y más terapia de reposición a base de cristaloides. Se mantuvo hospitalizado una semana, retirando el taponamiento sin presentar sangrado, por lo que se decidió alta hospitalaria.

En el mes de abril del 2017, desarrolló epistaxis izquierda y derecha siendo controlado en el servicio de Emergencias del Centro Hospitalario en la ciudad de Quito, Ecuador, con taponamiento nasal anterior, cohibido el evento hemorrágico prontamente y sin alteración de fórmula sanguínea; se mantuvo en observación 48 horas y su facultativo estableció su alta, se retiró de taponamiento en una semana sin repetir

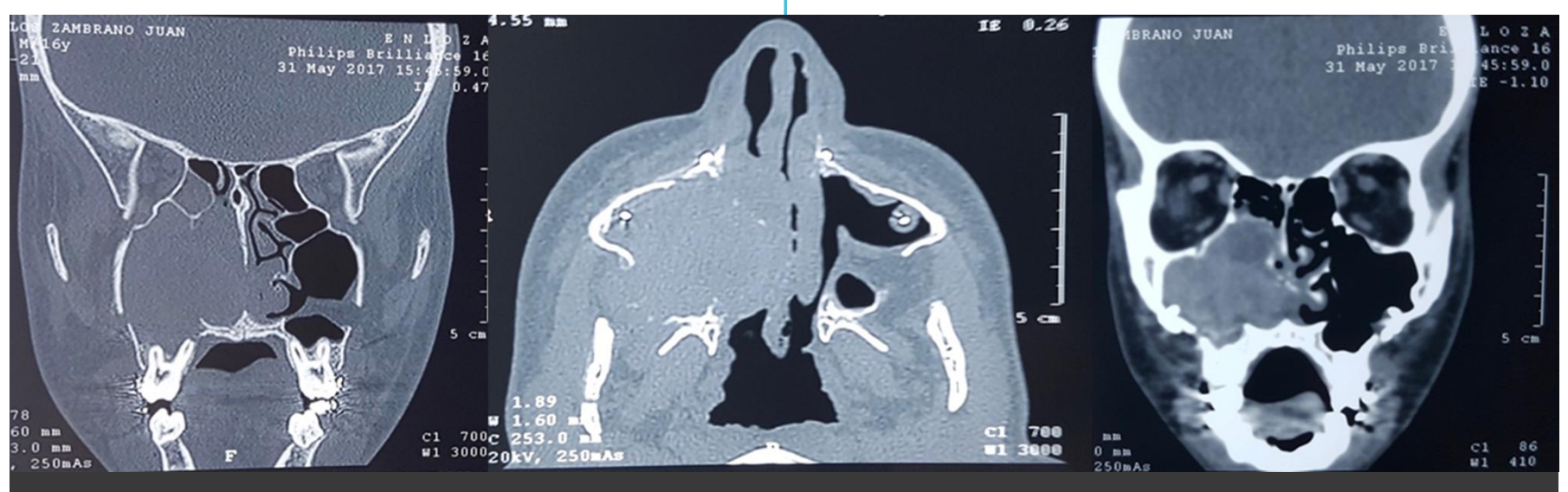

FIGURA 1. TC DE CRÁNEO 8 SEMANAS POSTERIORES A CIRUGÍA ORTOGNÁTICA 


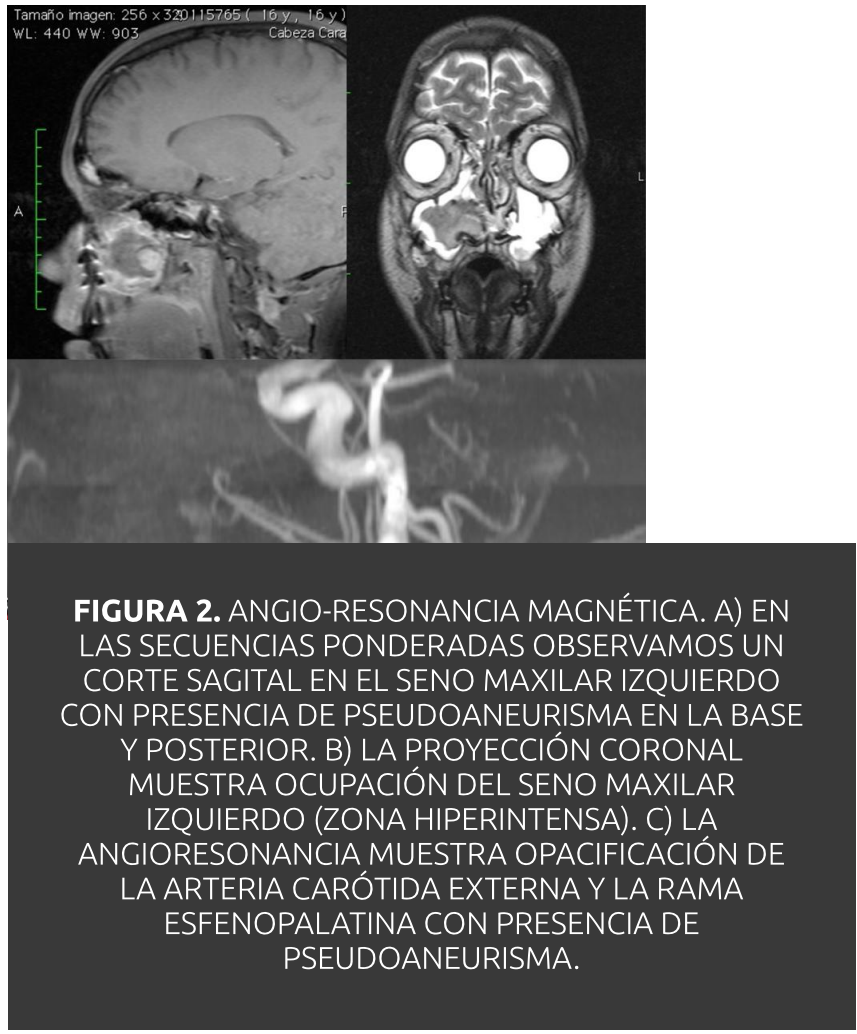

epistaxis. El mismo facultativo a cargo, le indicó una tomografía computarizada de macizo facial sin evidencia de entidad patológica, únicamente cambios propios del evento quirúrgico sin otra patología. En el mes de mayo se repite evento hemorrágico de fosa nasal izquierda principalmente; acude a Centro Hospitalario donde realizan las mismas maniobras para epistaxis (taponamiento nasal anterior y posterior).

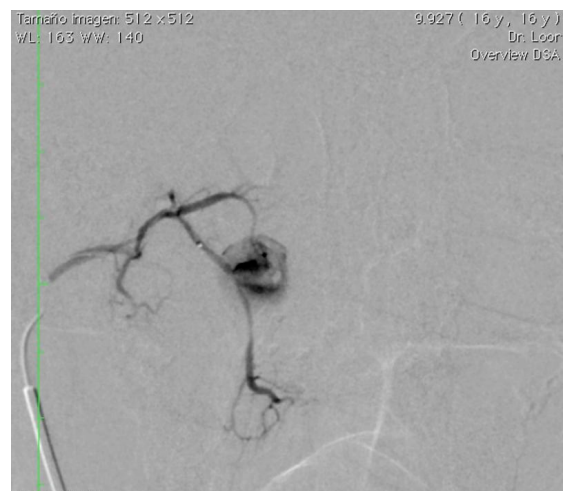

En Junio de 2017, el Cirujano Maxilofacial que realizó la Cirugía Ortognática se contactó al Instituto de Cirugía Maxilofacial de Guayaquil pidiendo revisar al paciente por nuevo evento hemorrágico, se ordenó su internamiento de Urgencia en OMNI Hospital, por Epistaxis masiva derecha, teniendo gasto de 700 cc, taquicárdico, hipotenso, se le coloca taponamiento nasal anterior y posterior derecho, interconsulta a Medicina Interna y Hematología por sospecha de presencia de inhibidores contra los factores de la coagulación por antecedentes de Tuberculosis. Son transfundidos paquetes de concentrados globulares y soluciones cristaloides. Es solicitada una tomografía del macizo facial (figura 1), donde se aprecia imagen hipodensa que ocupa el seno maxilar derecho, desplazamiento de septum nasal y cornetes derechos, sugerente de proceso neoplásico (Angiofibroma Nasofaringeo Juvenil) ó de restos de posible empaquetamiento transoperatorio.

Por indicación de Médico Radiólogo se realizó Angio-Resonancia de Senos Paranasales contrastada (figura 2, A), donde se reporta el velamiento del seno maxilar derecho de aspecto hemático, descartándose una neoplasia sinusal; luego de administrado el medio de contraste se sugiere como impresión diagnóstica pseudoaneurisma traumático. Ante ésta evidencia imagenológica, el Médico Radiólogo indicó la realización de Arteriografía, el día 16 de junio de

FIGURA 3. ANGIOGRAFÍA CON PSEUDOANEURISMA ARTERIA ESFENOPALATINA DERECHA. SE OBSERVA EL CATÉTER GUÍA Y EL MICROCATETER EMERGIENDO DEL MISMO, ESTE LLEGA A UNOS MILÍMETROS DEL PSEDOANEURISMA, PREVIO A LA LIBERACIÓN DE COILS. 


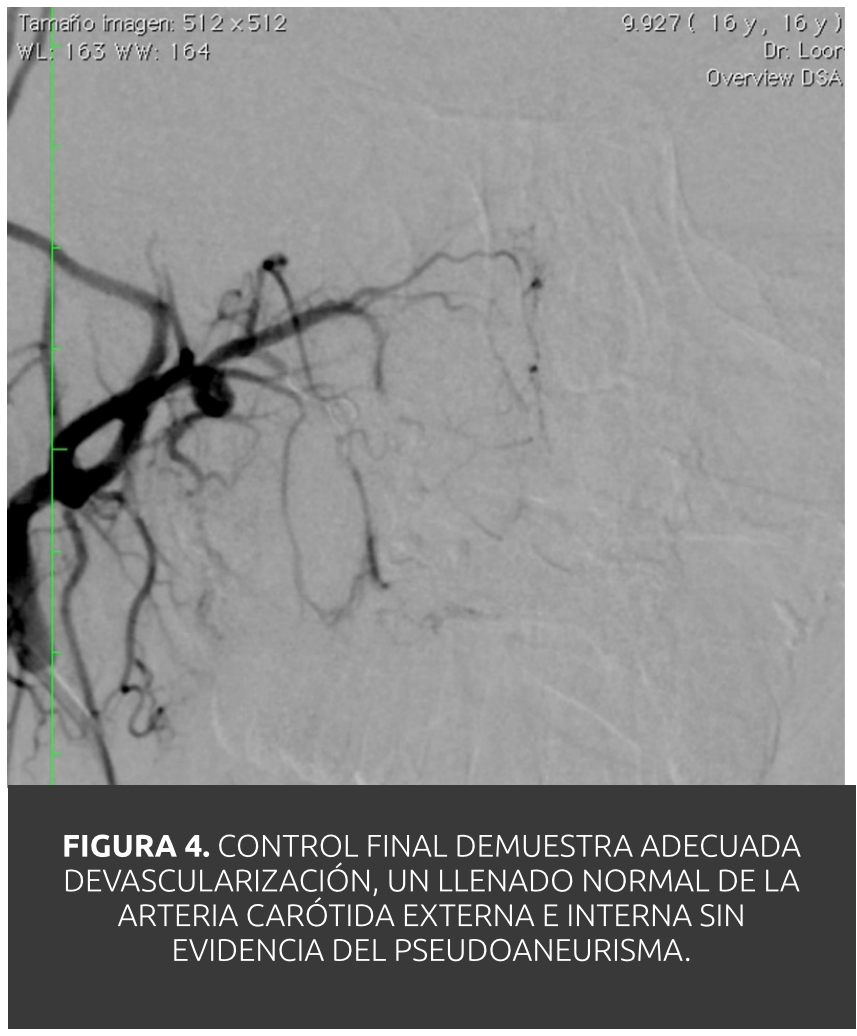

2017 (3 meses posterior a la cirugía Ortognática) encontrando un pseudoaneurisma de arteria esfenopalatina medial derecha (figura 2, B y C), se intentó realizar embolización sin embargo, ocurre vaso espasmo severo por lo que no se completa el procedimiento planificado.

Se esperó 48 horas, a la disminución del vasoespasmo y se procedió a la realización de arteriografía de ambas carótidas + embolización de arteria esfenopalatina medial derecha, introduciendo microcateter Headway Coating (MicroVention, Inc, CA, USA), soportado por un alambre guía $0.035 \times 150 \mathrm{~cm}$ procediendo a realizar microcateterismo con cable liberador de coil handheld, liberándose dos coils de las siguientes medidas: un coil $1 \mathrm{~mm} \times 1 \mathrm{~cm}$ barricade helical finish (Blockade Medical, CA, USA) y un coil $3 \mathrm{~mm} \times 4 \mathrm{~cm}$ barricade helical finish (Blockade Medical, CA, USA), logrando devascularización deseada. (figura 3 y 4).

Se realizó, en el mismo evento, arteriografía de arteria carótida izquierda y no se observan signos de sangrado ni lesiones en la línea media facial (figura 6). Es egresado 2 días posteriores es dado de alta sin complicaciones.

Acudió a cita de revisión un año posterior al primer evento, sin presentar datos de sangrado.

\section{Discusión}

La osteotomía Lefort es una técnica ampliamente utilizada mundialmente para la corrección de deformidades dento-esqueletales que afectan el tercio medio facial, algunas veces se usa para el abordaje a tumores de base de cráneo. Es considerada una técnica segura y con buenos resultados intra y posoperatorios. Los casos reportados de pseudoaneurismas posterior a cirugía en maxilar, están en relación a la arteria maxilar, en específico al ramo esfenopalatino 4.

Los aneurismas y pseudoaneurimas en la región de cabeza y cuello son poco frecuentes, en especial de la carótida externa. ${ }^{3}$

El tratamiento reportado comúnmente en la literatura es el tratamiento quirúrgico, sin embargo, se asocia a una mayor taza de morbilidad y mortalidad, debido sangrado profuso, dificultad de la exposición quirúrgica,

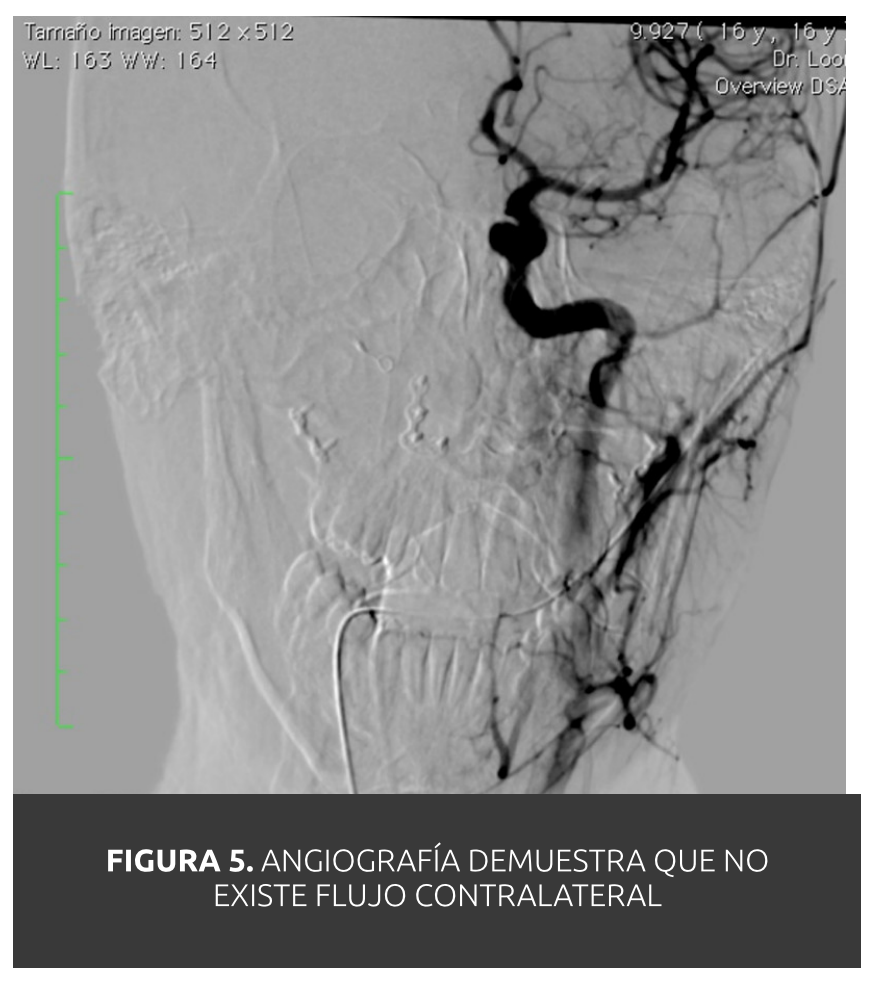


recurrencia de la lesión, necesidad de intervenciones múltiples sin la resolución completa y complicaciones cerebrales ${ }^{9}$.

La exploración quirúrgica urgente está indicada principalmente en casos de injurias cervicales penetrantes ${ }^{\mathbf{1 4}, \mathbf{1 6}}$.

El tratamiento endovascular debe ser considerado en casos daños vasculares no penetrantes, como un método seguro, ha sido usado por más de 45 años, en diferentes sitios anatómicos, como terapia para la oclusión mecánica, sin embargo, en la región maxilofacial rara vez se han implementado. Este embolo utiliza un agente temporal o permanente, como coils, partículas, stent cubierto, espuma, micro esferas o cuentas. Tiene como potencial complicación perforación de los vasos, alergia al material, vasoespasmos con fallo de la completa embolización, isquemia o daño a estructuras nerviosas, movimiento del material de oclusión, migración del coils, los balones se pueden desinflar, complicaciones neurológicas sin embargo los beneficios superan los riesgos. ${ }^{16}$

\section{Conclusión}

Los tratamientos tradicionales como el conservador o la exploración quirúrgica producen resultados insidiosos como sangrado abundante, daño a estructuras nerviosas, isquemia o incluso muerte. El caso mencionado podría ser como consecuencia de un down fracture muy agresivo, uso incorrecto de cinceles, alteración anatómica previa, manejo incorrecto de tejidos blandos, en fin, solo especulaciones.

El conocimiento del trabajo de la angiografía permite resultados favorables mientras el cirujano y el radiólogo intervencionista mantengan una comunicación adecuada.

El manejo de la radiología intervencionista como alternativa para el tratamiento de pseudoaneurismas tiene ventajas y podría ser considerado como el más recomendado para éstas patologías vasculares, sobre todo por el sitio anatómico de localización.

El presente caso, tuvo una resolución completa al tratamiento endovascular, sin datos de reincidencia a un año del evento quirúrgico inicial. Una examinación clínica minuciosa y una apropiada imagen radiográfica puede establecer un diagnóstico posible. Recomendamos considerar la embolización trans-arterial como primera línea de tratamiento para casos similares.

\section{Bibliografía}

1. Cunningham, L.; Van Sickels, J. y Brandt, M. Angioghapic Evaluation of the Head and Neck. Atlas of the Oral and Maxillofacial Surgery Clinics North America. 2003; 11(1) 73-86.

2. Carmo Galindo, C; Augusto Lima C; Galindo Filho, G y Marcondes Penha F. True atherosclerotic aneurysm of the facial artery. International Journal of Oral and Maxillofacial Surgery. 2006; 35 (6) 566-568.

3. Corvino A.; Catalano O.; Corvino F.; Sandomenico F.; Setola, S.; and Petrillo. A. Superficial temporal artery pseudoaneurysm: what is the role of ultrasound?. Journal Ultrasonographic. 2016; 19 (3) 197-201.

4. Pappa, H; Richardson D and Niven S. False Aneurysm of the Facial Artery as Complication of Sagittal Split Osteotomy. Journal of Cranio-Maxillofacial Surgery. 2008; 36 (3) 180-182.

5. Pourdanesh, F; Salehian $M$; Dehghan, $P$; Dehghani, $N$ and Dehghani, $S$. Pseudoaneurysm of the Superficial Temporal Artery Following Penetrating Trauma. The Journal of Craniofacial Surgery. 2013; 24 (4) 334-337. 
6. Silva A; O'Ryan F; Beckley M; Young $\mathrm{H}$ and Poor D. Pseudoaneurysm of a branch of the maxillary artery following mandibular sagittal split ramus osteotomy: case report and literature review, Journal of Oral and Maxillofacial Surgery. 2007; 65(9) 18071816.

7. Morken $\mathrm{M}$; Cappelen Johan; Kvistad K; Sovik $E$ and Stenseath $K$. Acute Endovascular Repair of latrogenic Rigth Internal Carotid Arterial Laceration. Acta Radiologica Short Reports. 2013; 2 (5) 1-3.

8. Chirichella T; Little J and Gruber B. Lingual Artery Pseudoaneurysm with Arteriovenous Fistula Formation Following a Gun Shot Wound. Journal Surgery Radiology. 2011;1(2)166-169.

9. Elton $V_{i}$ Turnbull $I$ and Foster M. An Overview of the Management of Pseudoaneurism of the Maxillary Artery: A Report of a Case Following Mandibular Subcondylar Osteotomy. Journal of Craniomaxillofacial Surgery. 2007; 35(1) 52-56.

10. Masella P; Megan H; Hall B; Verghese J. and Kellicut D. Posttraumatic Lingual Artery Pseudoaneurysm Treated with Ultrasound- Guided Percutaneous Thrombin Injection. Annals Vascular Surgerу. 2014; 28 (5) 1317-1322.

11. Osborne, P.; Sverzut T; Trivellato, A; Matias, C; Sverzut C and Sverzut, A. Carotid Jugular Fistula after Le Fort I Osteotomy. International Journal of Oral \& Maxillofacial Surgery. 2017; 46(7) 845-850.

12. Fonseca, Raymond J. Oral and Maxillofcial Surgery. Volumen III. Volumen Editor: Timothy A. Turvey. Second Edition. El Sevier Saunders. 2009 pp 185-187
13. Múnera, F.; Soto J.; Palacio, D; Velez $S$ and Medina, E. Diagnosis of Arterial Injuries Caused by Penetrating Trauma to the Neck: Comparison of Helical CT Angiography and Conventional Angiography. Radiology 2000; 216 (2) 356-362.

14. Lucas O; Naseem H-u-R, Davies J; Reynold R. and Bass K. Endovascular treatment of a carotid artery pseudoaneurysm due to penetrating trauma in a pediatric patient, Journal of Pediatric Surgery Case Reports. 2017; 19 19-21.

15. Serio, S. and Beeman, B. Delayed Presentation of a Common Carotid Pseudo-aneurysm Following Penetrating Trauma with Retrograde open Carotid Stent graft (ROCS). Annals of Vascular Surgery. 2014; 28 (7) 1799-1804.

16. Goksu, E; Senay, E and Aksoy C. Superficial temporal artery pseudoaneurysm: ultrasonographic diagnosis in the ED. American Journal of Emergency Medicine. 2009; 27 (5) 627-630.

17. Noy, D; Rachmiel, A; Emodi, O; Amsalem, Y; Israel, Y. and Nagler, R. Transarterial Embolization in Maxillofacial Intractable Potentially Life-Threatening Hemorrhage. Journal Oral Maxillofacial Surgery. 2017; 75(6) 1223-1231.

18. Shu-jun, C; Ning, J.; Yu-xuan, C, Xiao-xian, $H_{\text {.; }}$ Xue-mei, L.; Yan-kun, $L$ and Xiaozong,W. A Clinical Study of Large Cervical Vascular Injury in Penetrating Neck Trauma: Report of 6 Cases. China Journal of Oral and Maxillofacial Surgery. 2011: 9(2) 138-142. 